\title{
Development and verification of T-TRACE/PANTHER coupled code
}

\author{
A. Abarca ${ }^{1}$, M. Avramova ${ }^{1}$, K. Ivanov ${ }^{1}$, S. Verdebout ${ }^{2}$, D. De Meyer ${ }^{2}$, \\ C. R. Schneidesch ${ }^{2}$
}

${ }^{1}$ North Carolina State University, Raleigh, NC 27695

${ }^{2}$ Tractebel ENGIE

Boulevard Simon Bolivar 34-36 1000 Brussels, Belgium

aabarca@ncsu.edu,mnavramo@ncsu.edu,knivanov@ncsu.edu, christophe.schneidesch@tractebel.engie.com

\begin{abstract}
Multi-physics coupled simulations have become increasingly important during the last two decades being one of the major field of application in the nuclear technology. The nuclear reactors themselves are complex systems whose responses are driven by interactions between neutron kinetics, thermal-hydraulics, heat transfer, mechanics and chemistry. Probably, in a nuclear system, the most complex and important feedback effect takes place between the core neutron kinetics and thermal-hydraulics. The development of coupled thermal-hydraulic -neutron kinetics codes is a recurrent field of research for the nuclear industry.

This contribution, developed in the Consortium for Nuclear Power (CNP) framework, has the objective of develop a dynamic coupling, using TCP/IP based socket communication, between the thermal-hydraulic system code T-TRACE, Tractebel-ENGIE version of the latest US NRC TRACE release, and the multi-group 3-D nodal diffusion and core physics code PANTHER, developed and maintained by EDF Energy (UK). As a first step of the development, a fully temporally explicit coupling scheme has been developed between TRACE and PANTHER based on a boundary conditions exchange at the core level at each temporal iteration. The OECD TMI MSLB benchmark has been selected as verification scenario for testing the ongoing developing T-TRACE/PANTHER coupled code. The developed coupled code is benchmarked code-to-code against TRACE/PARCS and TRELAP5/PANTHER.
\end{abstract}

KEYWORDS: TRACE, PANTHER, coupled codes, socket communication, TMI MSLB.

\section{INTRODUCTION}

Tractebel Engineering (TE) has been working since 2000 on the application of multi-physics code packages in order to perform realistic simulation of PWR reactor transients. The objective is to provide tools and methods for independent verification of the safety analyses, in particular those performed for the Belgian plants. The TE code multi-physics package based on the coupling of the 3D neutron code PANTHER and the system code RELAP was first used in the Main Steam Line Break in Hot Zero Power conditions (MSLBHZP) accident analysis methodology [1]. This methodology has been accepted by the Belgian Safety Authorities for applications related to the power uprate and steam generator replacement project of the Doel 1 and Doel 2 plants, to the Tihange-3 FSAR re-analysis and to justify the cycle length extension of Doel 4 to 18 months. Those applications were extended to accident analysis methodologies at hot full power 
condition like the MSLB-HFP and the Feed Water Line Break (FWLB) accident [2] [3]. Their application to design extension conditions analyses in the framework of the implementation of the WENRA Safety Reference Levels [4] for Doel 4 and Tihange3 is currently under review.

The capacities of the TE multi-physics code packages are extended with the inclusion of the TRACE code which is the latest in a series of advanced, best-estimate reactor systems codes developed by the U.S. NRC.

\section{The TE multi-physics code package}

\subsection{Description}

The TE multi-physics code package (Fig. 1) is composed by the 3-dimensional neutronic code, PANTHER, with a 2-way dynamic coupling to the system thermal hydraulic code T-RELAP5 (via the TALINK coupling supervisor, presented in more detail in the next section) and to the core thermal hydraulic code COBRA-3C_TE (using sockets). One-way links provide time-dependent realistic power histories and distributions for specific criteria evaluation, either with the sub-channel thermal hydraulic code COBRA$3 \mathrm{C}$ or with the fuel rod thermal-mechanical code FRAPTRAN.

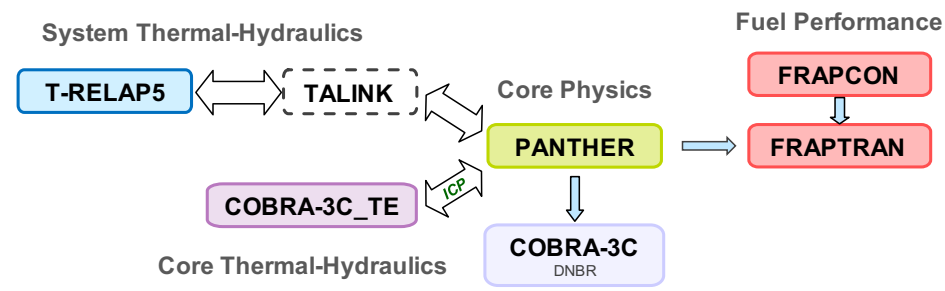

Figure 1. The TE multi-physics code package.

PANTHER [5] is a multi-energy groups 3-D nodal diffusion code for both steady-state and transient core simulation. PANTHER developed and maintained by EDF Energy (UK) to perform a complete range of PWR reactor calculations for fuel management design safety parameters assessment, fault transient analysis and operational support. A built-in LWR thermal-hydraulics module determines the variation of local variables having a significant feedback on core neutronic properties. This module is based on the model of T-H channels in isolation where fuel rod segments serve as heat structures surrounded by a representative quantity of coolant under forced convective flow conditions from core inlet to outlet.

The RELAP5 code [6] is a widely used best estimate system thermal hydraulic transient analysis code, developed by the USNRC, to simulate the LWR plant response for different postulated accidents including loss of coolant and reactivity insertion accidents as well as operational transients.

T-RELAP5 is a version of RELAP5 which has been modified in TE to incorporate FORTRAN routines allowing coupled data transfer with TALINK compatible with PANTHER data structure.

COBRA-3C_TE [7] is a TE version of the sub-channel thermal hydraulic code COBRA3C_MIT2 code reengineered to be coupled to PANTHER in order to replace and extend the PANTHER LWR module modelling capacities to channel cross flows, turbulent mixing and wider flow-regimes transitions.

FRAPTRAN [8] code is developed by Pacific Northwest National Laboratory (PNNL) for analysing the thermal-mechanical behaviour of a LWR fuel rod under transient and accident conditions such as LOCA and RIA. It is used on the basis of the steady-state fuel conditions calculated by the FRAPCON code. 


\subsection{Coupling 3D neutron kinetics to system thermal-hydraulics}

The current T-RELAP5/PANTHER coupling is a traditional external 2-ways dynamic coupling where the TALINK interface controls the data transfers between the two processes which exchange boundary conditions using temporary data files. TALINK also performs, on user's request, data processing at each step between reading in and writing out exchanged data. This traditional approach preserves the numerical properties of the solution algorithms implemented in each code, which thus keeps its own time integration scheme. Such approach simplifies code installation and quality assurance procedures as each code is used in its usual standalone application configuration and environment.

The two codes are coupled at the core level using a split-vessel model where on the system side, the reactor vessel (downcomer, lower plenum, core and upper plenum) is split into several regions, each one of those being associated with one steam generator loop.

The TMI OECD/NEA PWR MSLB benchmark problem [9] is used as a demonstration case in this paper. This benchmark is based on real plant design and operational data of the Three Mile Island - Unit 1 Nuclear Power Plant, characterised by a B\&W design 2-loop reactor with 177 fuel assemblies. The main scenario for this MSLB accident assumes a double-ended break to occur in Hot Full Power at End-of-Cycle conditions in one main steam pipe, upstream of the main steam isolation valves followed by reactor trip due to either low reactor coolant pressure or high neutron flux.

In this 2-loop reactor case, the core model has therefore two equal regions (Fig. 2). On the system side, the RELAP core consists in two parallel core channels that communicate fluid only at the inlet and outlet plena. Each core channel contains 12 equal flow volumes with heating structures and receives heat from the corresponding PANTHER half-core set of fuel assemblies. The heat generated in each of the 24 layers is re-mapped within to match the axial nodalization.

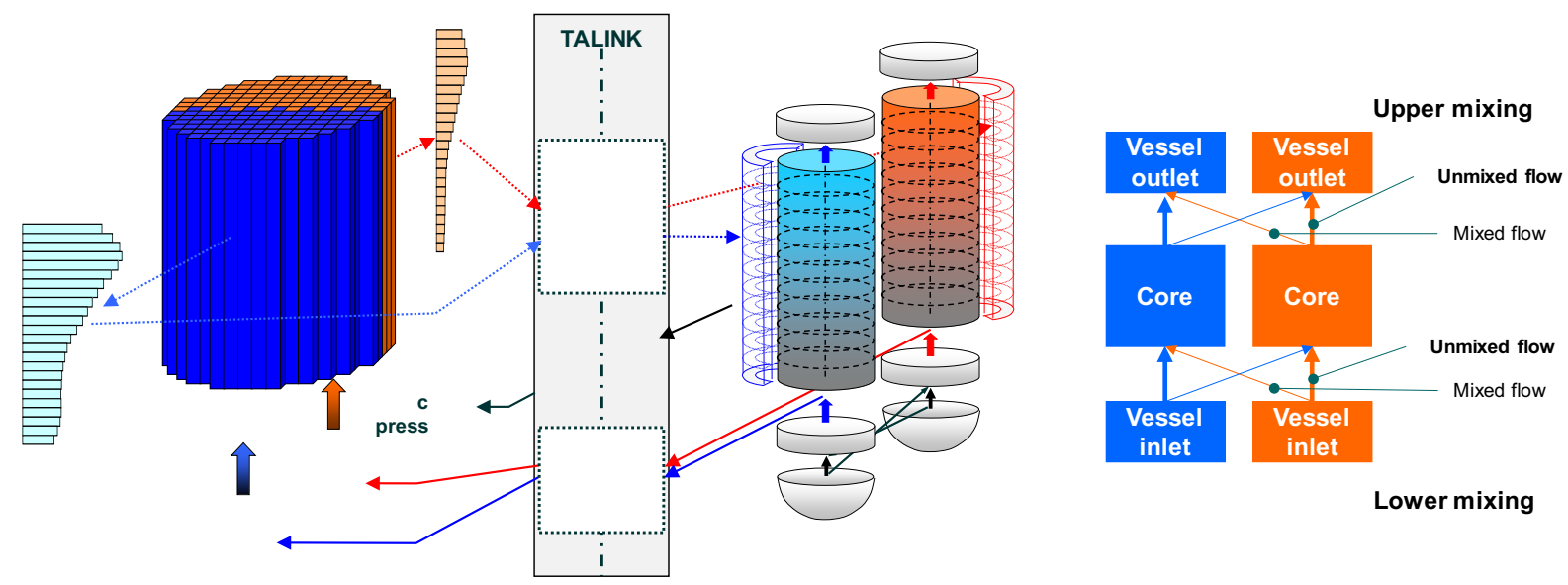

Figure 2. Schematic coupling between core neutronics and system T-H.

This coupling scheme takes benefit of the full-core T-H and fuel rod calculations by PANTHER LWR module and uses by construction two separate "core T-H" models. However, as also by construction, the two cores share identical boundary conditions, the sector-wise coolant enthalpies at core outlets are practically identical but for minor discrepancies due steam-tables or correlation differences. 
The core inlet conditions applied to 3D neutron kinetics are calculated by :

- Temperature per sector $\left(T_{-}\right.$): from sector temperatures at the inlet of each core volume;

- Channel mass flow in each sector (W_): from coolant mass flow at the inlet of each core volume converted into corresponding channel-wise mass flow as required by ;

- Reactor pressure (P): from mean pressure axially averaged in split core volumes applied. It is applied as system pressure since no pressure drops are calculated in .

The core boundary conditions for each time step are computed by :

- Nuclear power profile per sector $(\mathrm{Pz})$ : the total nuclear power profile in each sector is axially distributed on the heat structures for heat generation in each split core volume;

- Scram time: neutron flux is evaluated in and compared with high flux setpoint. The time of high flux detection is transferred to to activate the code I\&C system models.

Mixing between half core segments takes place at the inlet and outlet of the core (lower and upper plenums) and also by means of the significant core bypass flow through the upper head. The desired mixing ratio was achieved by adjusting cross sections and friction factors in the connecting upper and lower plenum junctions. There is a single guide tube connecting the upper plenum and the upper head. This feature introduces mixing flow between the two core halves, which is in addition to any mixing in the upper and lower plenum.

\subsection{Extending coupling capacities}

The capacities of the TE multi-physics code packages are extended with the inclusion of TRACE code [10], which is the latest in a series of advanced, best-estimate reactor systems codes developed by the U.S. NRC. TRACE is the product of a long term effort to combine the capabilities of the NRC's four main systems codes (TRAC-P, TRAC-B, RELAP5 and RAMONA) into one modernized computational tool.

Coupling TRACE to PANTHER follows the same principles as T-RELAP5/PANTHER coupling but the TALINK data file exchange between the two codes is replaced by direct data exchange protocols.

PANTHER code offers the possibility to exchange data with other active processes by sending and receiving records through a socket connection without any need of source code modification. The IPC (Inter Process Communications) implemented in PANTHER is based on the TCP/IP protocols as set up in Unix via "Internet Domain" sockets. When the sockets are used to exchange data with PANTHER and the other code (for example TRACE) does not include compatible IPC, its source has to be modified by adding or adapting subroutines to handle the data exchange of PANTHER record-components with sockets IPC. This TCP/IP based socket communication is widely used in TE to link PANTHER to other codes.

The TRACE source code was modified by NCSU to include a PANTHER coupling option by programming the FORTRAN sub-routines as implemented in COBRA for sockets coupling with PANTHER.

Two different ways to achieve the external T-TRACE/PANTHER coupling are considered:

- Duplicating the coupling principles of the T-RELAP5/PANTHER as where boundary conditions between the T-TRACE (split) vessel core model and the PANTHER core are exchanged, keeping the core TH feedbacks calculated on the PANTHER side by its LWR thermal hydraulics function or by external COBRA coupling (Fig. 2);

- Applying the coupling principles in TRACE-PARCS where only the neutronics would be solved in PANTHER keeping all (T-H) feedback variables calculated in T-TRACE 3D core and transferred after suitable mapping to PANTHER as 3D-distributions for lumped assembly subsets. 
The coupling principles of the T-RELAP5/PANTHER are in a first stage developed in T-TRACE for consistency with the other components of TE's package and existing methodologies.

\section{THE TRACE PANTHER COUPLING}

The T-TRACE/PANTHER coupling is developed in the Consortium for Nuclear Power (CNP) framework on the basis of a dynamic coupling scheme. The coupling algorithm has been developed using a Unix socket interface based on the IPC used in TE to link PANTHER to other codes, allowing communication between TRACE and PANTHER without modifications in the sources of PANTHER.

\subsection{Coupling algorithm}

A Fortran wrapper for the $\mathrm{C}$ based Unix socket functions has been developed and added to the TRACE compilation project. This wrapper is included in a single Fortran module (SocketComm module) in the sources of TRACE and it is included, as a static library, in the main compilation project. The compilation and building systems of TRACE were slightly modified to include the socket library interface.

The developed coupling algorithm reproduces the T-RELAP5/PANTHER external coupling that was presented in the section 2. In summary, the boundary conditions between the split vessel core model in TRACE system code and the PANTHER core are exchanged, keeping the core T-H feedbacks calculated by PANTHER. The analyst is able to activate the coupling procedure between TRACE and PANTHER by a command line argument (--panther) added to TRACE.

The synchronization and communication points between the coupled codes were identified and implemented in TRACE. TRACE runtime flow diagram (Fig. 3) shows the implemented synchronization calls and information exchange. At the very beginning of the simulation TRACE reads the command line arguments that may contain the argument to set the TRACE-PANTHER coupling algorithm.

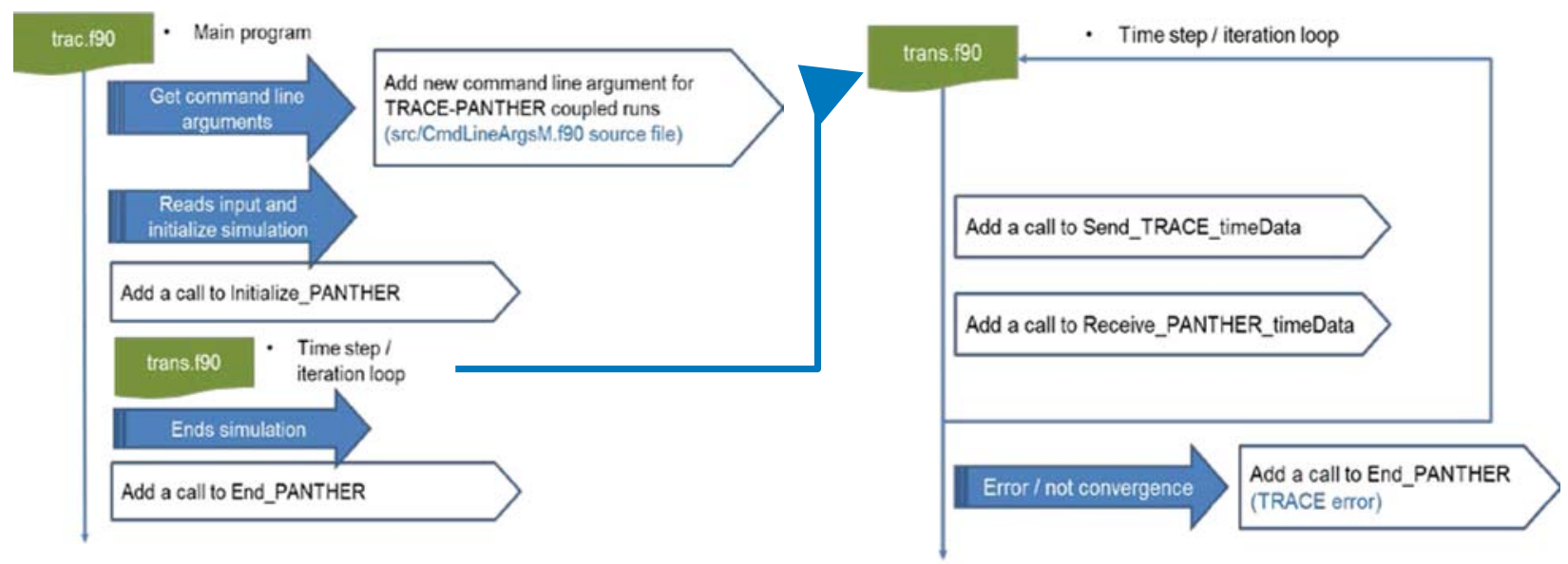

Figure 3. Schematic coupling between TRACE and PANTHER.

To address the differences between the nodalization of thermal-hydraulic and the neutronic models, an external mapping file has to be included as an input of the coupled code. The use of different $\mathrm{T}-\mathrm{H}$ and $\mathrm{N}$ $\mathrm{K}$ meshes is justified by the different approaches used in each code for the description of the real geometry of the system. This problem is generally overcome defining a mapping between both nodalizations. As was pointed before, for this coupling a mapping file has to be mandatorily included as an input to define the 
correspondence between azimuthal sectors in the PANTHER model and component numbers in TRACE (vessel, pipes, or channels).

\subsection{TMI TRACE split-vessel model}

TMI OECD MSLB benchmark being chosen to verify the T-TRACE/PANTHER coupling, the original cartesian TRACE vessel model has been modified to obtain a split-vessel model equivalent to the TRELAP5 model presented in the Section 2. The nodalization of the vessel reproduces the same as where boundary conditions between the T-TRACE (split) vessel core model and the PANTHER core are exchanged.

On the T-H side, the TRACE split-vessel model consists in two parallel active flow channels with its respective bypasses that communicate fluid only at the inlet and outlet plenum using single junction components (Fig. 4). The split-vessel model also includes two downcomer regions driving the coolant from the cold leg to the lower plenum. Axially, the two flow channels and the bypasses has been divided into 12 equally spaced nodes with heat structures to represent the heat transfer from the corresponding PANTHER half-core. The two primary and secondary coolant loops, as well as the reactor control system, remain unaltered from the original model.
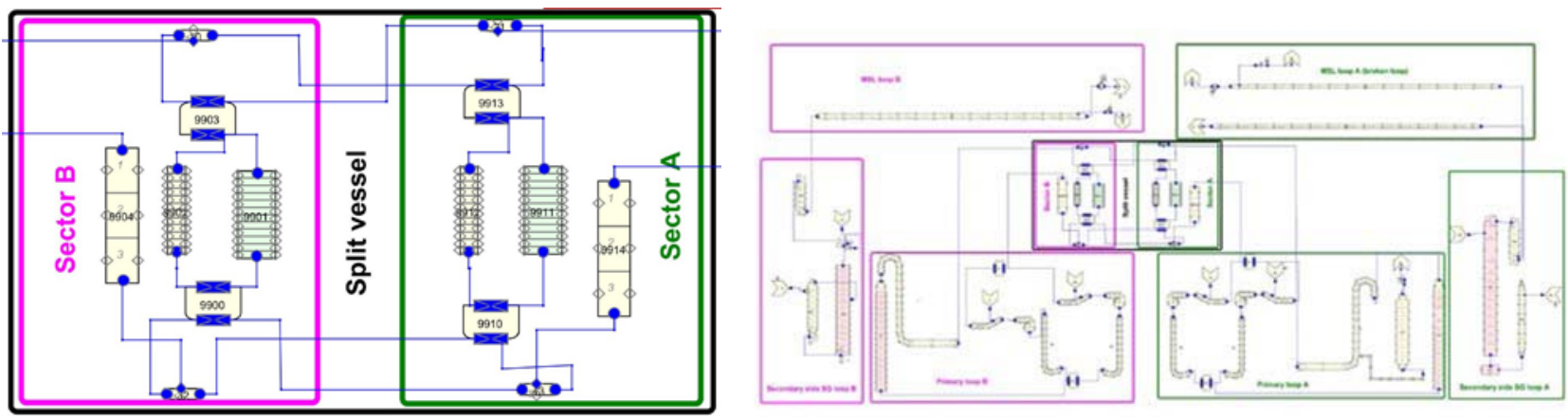

Figure 4. TRACE split-vessel model for OECD/NRC TMI MSLB benchmark.

\section{RESULTS AND DISCUSSION}

The OECD MSLB benchmark exercise 3 is chosen to test the T-TRACE/PANTHER coupling. TE has participated to this benchmark using the T-RELAP5.mod2.5 which was the version of the code approved for licensing activities [9] at the benchmark definition. Figure 5 compares the benchmark reference with mod2.5 and the current mod3.3 version which does not show at macroscopic level major discrepancies.

The T-TRACE/PANTHER coupling has been tested successfully on a stabilization phase as pseudotransient without any change in the boundary conditions, demonstrating that the data exchange using the sockets matches the specifications.

When applied to the OECD MSLB scenario, the coupled transient responses to the event differ between TTRACE/PANTHER and T-RELAP5/PANTHER (Fig. 5). Those discrepancies are not caused by the coupling process itself which performs adequately but are mostly due to a stronger cooling effect obtained by T-TRACE/PANTHER as illustrated by the comparison of the evolutions of the total power and the core inlet temperatures for each sector of the split-vessel model. 
This behaviour is explained by system model differences. The OECD MSLB T-TRACE input for this coupling demonstration has been developed from a simplified model where the metal structures of the primary circuit are not explicitly taken into account as in T-RELAP model, lacking therefore the thermal inertia which alleviates the cooling effect as shown in the T-RELAP/PANTHER transient. In addition, stand-alone comparisons on the two system models demonstrate a flow discharge through the break which differs between codes and leads to stronger overfilling and cooling in TRACE, resulting in an additional 'overcooling' of the primary side with respect to RELAP and benchmark.
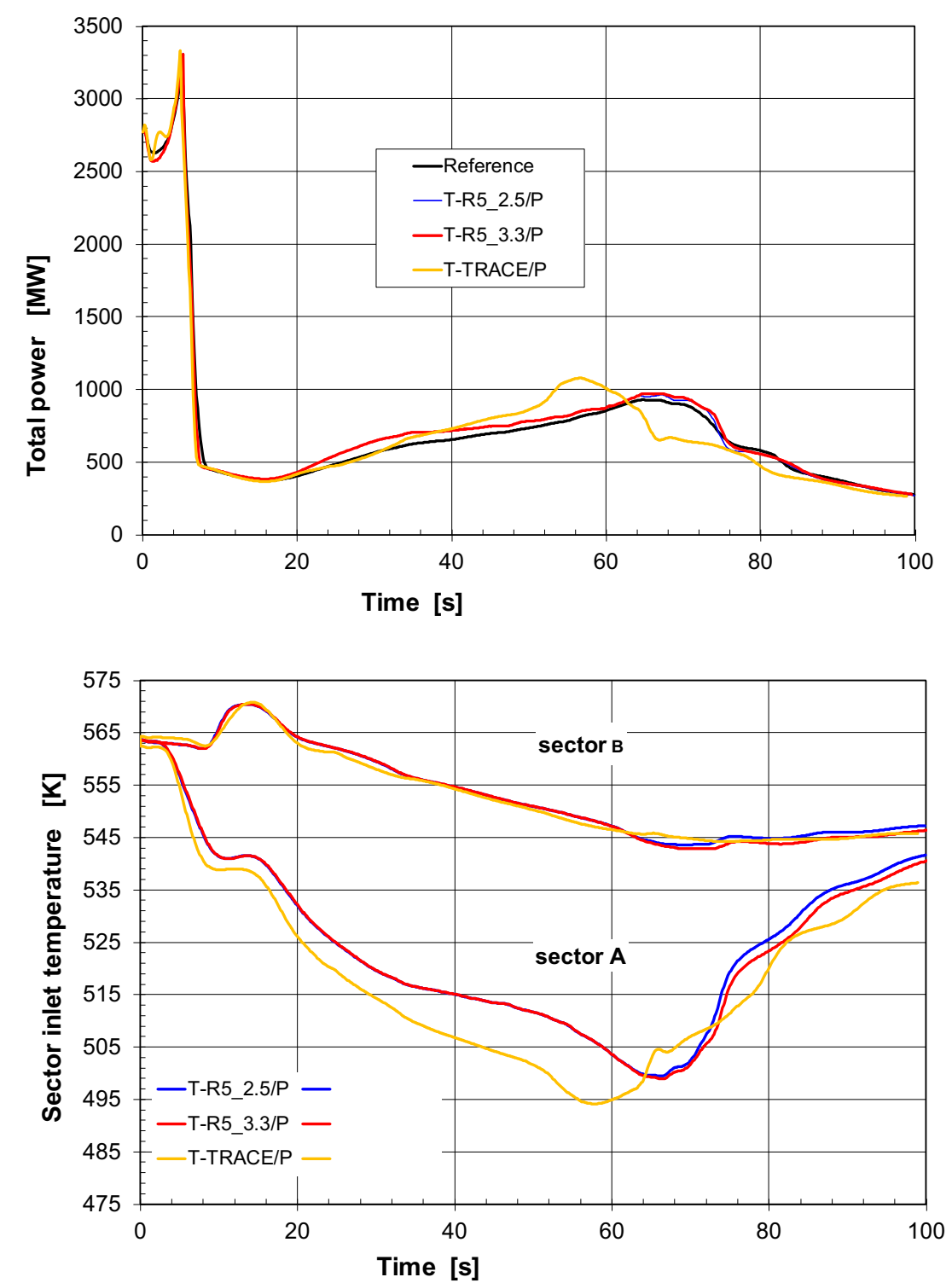

Figure 5. OECD MSLB benchmark exercise 3 transient results.

\section{CONCLUSIONS}

The T-TRACE/PANTHER coupling has been implemented successfully by dynamic external coupling using sockets through code modification for implementing the data exchange and data processing 
subroutines in the TRACE code sources. The sockets coupling capacity implemented in PANTHER is based on the TCP/IP protocols as set up in Unix via "Internet Domain" sockets.

The sockets implementation in this prototype coupling does not incorporate facilities to tailor the data exchange process without modifying the sources of the codes. They can transfer data but do not manipulate them. Therefore, once a set of data to send to or to be received has been implemented in the code, any additional variable to be exchanged requires another version of the code.

When applied to the demonstration case OECD MSLB benchmark, discrepancies between the predictions of RELAP and TRACE transient responses at system-level mostly reflect the differences in the modelling of the system components, in particular the metal structures. Further work on the convergence between the two system models will allow to produce a better agreement on the global transient behaviour. However, regardless of the different system responses, the coupling achieves rightfully the 2-ways exchange between the physics in each code.

The next stage of the development beyond this demonstration phase is to introduce more flexibility in the practical coupling capacities by using as much as possible the inherent coupling functionalities of all codes.

\section{ACKNOWLEDGMENTS}

The authors would like to acknowledge the contribution of the following experts at Tractebel: V. Jammot and A. Kovtovnyuk, who have contributed to this development.

\section{REFERENCES}

1. J. Zhang, et al, "Coupled RELAP5/PANTHER/COBRA Steam Line Break Accident Analysis in Support of Licensing Doel 2 Power Uprate and Steam Generator Replacement," Proceedings of International Meeting on Updates Best-Estimate Methods in Nuclear Installation Safety Analysis, Washington, D.C., USA (2004).

2. R. Van Parys, S. Bosso, C. R. Schneidesch and J. Zhang, "Analysis of the MSLB with LOOP using the fully coupled RELAP5/PANTHER/COBRA3C_TE code package", the 11th International Topical Meeting on Nuclear Reactor Thermal-Hydraulics (NURETH-11) Avignon, France (2005).

3. M. Haedens J. Zhang and C. R. Schneidesch, "Coupled RELAP5/PANTHER and COBRA Analysis of the Steam Line Break Accident Using Core Inlet Mixing Matrix Generated by CFD Calculations", Proceedings of the 9th International Topical Meeting on Nuclear Thermal-Hydraulics, Operation and Safety, (2012).

4. Western European Nuclear Regulators Association, "WENRA Safety Reference Levels for Existing Reactors", September 2014.

5. P. K. Hutt, N. Gaines, M. J. Halsall, M. McEllin, and R. J. White, "The UK Core Performance Code Package," Nucl. Energy 30, 291-298 (1991).

6. V.H. Ransom, et al., "Relap5/Mod2 Code Manual," NUREG/CR-4312, USNRC (1985).

7. J.W. Jackson and N.E. Todreas, "COBRA3C/MIT-2 - A Digital Computer Program For Steady State And Transient Thermal-Hydraulic Analysis Of Rod Bundle Nuclear Fuel Elements", Energy Laboratory Report No. MIT-EL 81-018, June 1981.

8. K. J. Geelhood and W.G. Luscher, J. M. Cuta, "FRAPTRAN-1.5: A Computer Code for the Transient Analysis of Oxide Fuel Rods", NUREG/CR-7023, Vol. 1, Revision 1, USNRC, May 2014.

9. C. R. Schneidesch and J. Zhang, "Simulation of the OECD main-steam-line-break benchmark exercise 3 using the coupled RELAP5/PANTHER codes", Nuclear Technology, 146, pp 1-14 (2004).

10. US NRC, "TRACE V5. 0 Theory Manual, Field Equations, Solution Methods, and Physical Models," tech. rep., US NRC (2008). 